\title{
A New Method of Blind Deconvolution for Colour Fundus Retinal Images
}

\author{
Bryan M. Williams ${ }^{1}$, Ke Chen ${ }^{1}$, Simon P. Harding ${ }^{2}$, and Yalin Zheng ${ }^{2}$ \\ 1 Department of Mathematical Sciences, University of Liverpool, Peach Street, \\ Liverpool, L69 3BX, UK \\ bryan@liv.ac.uk \\ Home page: http://www.liv.ac.uk/cmit \\ 2 Department of Eye and Vision Science, University of Liverpool, Daulby Street, \\ Liverpool, L69 3GA, UK \\ yzheng@liv.ac.uk
}

\begin{abstract}
Fundus retinal imaging is widely used in the diagnosis and management of eye disease. Blur commonly occurs in the acquisition and when it is severe the resulting loss of resolution hampers accurate clinical assessment. In this paper, we present a new technique to address this challenging problem. We make use of implicitly constrained image deblurring, which is known to provide improved results over unconstrained and explicitly constrained methods, and build this into a multi-channel variational framework for parametric deblurring. We propose a new method for automatically selecting the regularisation parameter in the absence of the true (sharp) image using vessel segmentation. We then modify the model to include a regularisation coefficient function which is dependent on an available image mask in order to avoid potential inaccuracies caused by the addition of artificial masks. We present experimental results to demonstrate the effectiveness of our new method.
\end{abstract}

Keywords: Semi-blind deconvolution, regularisation parameter selection, vessel segmentation, constrained deconvolution, fundus image

\section{Introduction}

Fundus retinal imaging is widely used in the diagnosis and management of eye disease, such as diabetic retinopathy and age-related macular degeneration. Blur commonly occurs in the acquisition due to various factors such as media opacity, refractive errors and involuntary eye movement of patients. When blur is severe, the resulting loss of resolution hampers accurate clinical assessment, which remains a major challenge for early disease detection, accurate diagnosis and outcome monitoring. In an existing program of diabetic retinopathy screening, approximately $5 \%$ of the images are too blurred for assessment. This necessitates the development of effective image deconvolution techniques. As a mathematical consideration, we model the problem of blur as a convolution $[\kappa * u](\mathbf{x})$ of a sharp image $u$ which we wish to recover and a blur function $\kappa$. We also consider the presence of noise which makes this an ill-posed and challenging problem.

X. Chen, M. K. Garvin, J. Liu, E. Trucco, Y. Xu (Eds.): OMIA 2015, Held in Conjunction with MICCAI 2015, Munich, Germany, Iowa Research Online, pp. 129-136, 2015. Available from: http://ir.uiowa.edu/omia/2015_Proceedings/2015/ 
Deconvolution problems may be classed as: 1) non-blind in the case of known blur, in which case the image is to be recovered; 2) blind in the case of unknown blur, which involves approximation of the unknown blur function; 3) semi-blind which includes problems where the blur function may be assumed to belong to a class of parametric functions.

In this paper, we develop a method for semi-blind deconvolution using implicitly constrained deblurring. Implicitly constrained deblurring methods are known to offer improved results over unconstrained and explicitly constrained methods, which typically involve thresholding. This new method allows for restoring images from out of focus blur degradation quickly by reducing the problem of identifying a large number of unknowns to one of estimating a few parameters. We also address the issue of deblurring given artificial masks surrounding the field of view which affect the quality of the restored image. We introduce a regularisation coefficient function which increases regularisation in these regions, thus reducing influence in the deconvolution problem. The selection of optimal regularisation parameters is an important consideration. For retinal images, we introduce a method of determining the regularisation parameter automatically by making use of vessel maps. The main contributions of this paper are:

1. to incorporate constraints implicitly into a parametric framework,

2. to introduce a regularisation coefficient function to allow for artificial masks, 3. to develop a method for selecting the degree of regularisation automatically.

This paper is organised as follows. In $\S 2$, we introduce some relevant image deconvolution techniques. In $\S 3$, we present the new model proposed in this paper. In $\S 4$, we show experimental results and in $\S 5$, we conclude this work.

\section{Image Deconvolution for Colour Fundus Imaging}

There exist many techniques for recovering an image from blur degradation such as that presented in [7]. In [3], the authors improved on [7] by proposing a total variation (TV) functional (1) due to its ability to recover edges and an alternate minimisation scheme which aims to find functions $u$ and $\kappa$ which minimise

$$
\mathcal{J}(u, \kappa) \equiv \frac{1}{2} \int_{\Omega}(\kappa * u-z)^{2} \mathrm{~d} \mathbf{x}+\alpha_{1} \int_{\Omega}|\nabla u|_{\beta_{1}} \mathrm{~d} \mathbf{x}+\alpha_{2} \int_{\Omega}|\nabla \kappa|_{\beta_{2}} \mathrm{~d} \mathbf{x}
$$

where $*$ denotes convolution, $\mathbf{x}=(x, y)$ for a 2-dimensional image, $\alpha_{1}$ and $\alpha_{2}$ are small positive parameters which measure the trade-off between data fitting and smoothness, and $|f|_{\beta}=\sqrt{|f|^{2}+\beta}$ is a smooth approximation to TV regularisation where $\beta$ is a small parameter used to avoid division by zero in the resulting Euler-Lagrange (EL) equations. In order to minimise the functional, we derive the first order optimality conditions obtained from the derivatives of $\mathcal{J}$ with respect to $u$ and $\kappa$ and solve them iteratively.

While unconstrained TV deblurring provides good results, the restored image may contain values significantly outside of the boundary of the intensity range meaning that it must be projected back onto the correct range. This is typically done in a naive way which can lead to a significant drop in the quality 
of the recovered image [2]. To address this, we implement a model for implicitly constrained deblurring which is known to provide a better result [4].

We introduce a transform of the image given by $\tau_{\mathbf{a}}(\psi)$ which has a range contained in $\mathcal{C} \subseteq \mathbb{R}^{m \times n \times 3}$ and where $\psi$ is a function such that $\tau_{\mathbf{a}}(\psi)$ is equal to the image $u$. The proposed bounded transform and its inverse are given by

$$
\tau_{\mathbf{a}}(\psi)=\frac{a_{1}+2 a_{4}}{1+a_{2} e^{-\frac{2 \psi}{a_{3}}}}-a_{4}, \quad \text { and } \quad \xi_{\mathbf{a}}(u)=\frac{-a_{3}}{2} \ln \frac{a_{1}-u+a_{4}}{a_{2}\left(u+a_{4}\right)},
$$

respectively such that $u=\tau_{\mathbf{a}}(\psi)$ and $\psi=\xi_{\mathbf{a}}(u)$ where the parameters $a_{1}, \ldots, a_{4}$ may be preselected and are problem-dependent. More details can be found in [4]. To give an example, for an image whose intensity values are contained in the range $[0,1]$, an appropriate parameter choice can be $\mathbf{a}=\left\{1,1,0.44,10^{-2}\right\}$. To avoid introducing non-linearity into the fitting term with the transform, the augmented Lagrangian method is used to drive $u$ and $\tau_{\mathbf{a}}(\psi)$ close to each other, with the latter remaining in the regularisation term. Similar considerations may be applied to the point spread function (psf) to form a constrained blind method.

In our new model, we adopt a parametric approach to kernel identification which relieves us from constraining the range of the blur function. There remains the problem of selecting the regularisation parameter which gives the optimal resulting image which we consider in the following section.

\section{A New Model for Parametric Constrained Deblurring with Automatic Regularisation Parameter Selection}

Here, we build on the previous section by developing a semi-blind framework for constrained multi-channel image deblurring and blur function identification.

\subsection{Enhancement I - Constrained Parametric Image Deblurring}

Many techniques for blind deconvolution involve blur function identification by the joint minimisation of a variational model in order to recover the blur function which minimises the functional. In this case, we assume that the blur is out of focus, which may be modelled by the Gaussian function

$$
\kappa_{\mathcal{G}}(x, y ; \sigma)=\left(2 \pi \sigma^{2}\right)^{-1} \exp \left(-x^{2}+y^{2} \sigma^{-2} / 2\right) .
$$

Parametric models are beneficial because they reduce the problem of identifying $m \times n$ unknowns to only a small set of parameters. We may substitute $\kappa_{\mathcal{G}}$ into the constrained problem which gives the new aim of finding the parameter $\sigma$ which minimises the resulting functional. Since the function $\kappa_{\mathcal{G}}$ is automatically constrained we do not need additional constraints given by $\tau_{\mathbf{b}}(\omega)$ and the smoothness term. Extending the problem to multi-channel rgb images so that 
the psf is influenced by each channel, we write the functional to be minimised as

$$
\begin{aligned}
& \max _{\varphi_{c}} \min _{u_{c}, \psi_{c}, \sigma}\left\{\mathcal{F}\left(u_{c}, \psi_{c}, \sigma ; \varphi_{c}\right)=\sum_{c \in\{r, g, b\}} \frac{1}{2}\left\|\kappa_{\mathcal{G}}(\sigma) * u_{c}-z_{c}\right\|_{L^{2}(\Omega)}^{2}\right. \\
& \left.+\alpha \int_{\Omega}\left|\nabla \tau_{\mathbf{a}}\left(\psi_{c}\right)\right| \mathrm{d} \mathbf{x}+\frac{\gamma_{c}}{2}\left\|u_{c}-\tau_{\mathbf{a}}\left(\psi_{c}\right)\right\|_{L^{2}(\Omega)}^{2}+<\varphi_{c}, u_{c}-\tau_{\mathbf{a}}\left(\psi_{c}\right)>\right\} .
\end{aligned}
$$

We minimise this functional by deriving the EL equations given below and finding the arguments which allow them to be equal to zero.

$$
\begin{aligned}
\mathcal{F}_{1}\left(\sigma, u_{c}, z_{c}, \psi_{c}, \varphi_{c}\right) & =\kappa_{\mathcal{G}}^{\dagger}(\sigma) *\left(\kappa_{\mathcal{G}}(\sigma) * u_{c}-z_{c}\right)+\gamma\left(u_{c}-\tau_{\mathbf{a}}\left(\psi_{c}\right)\right)+\varphi_{c}^{\top}, \\
\mathcal{F}_{2}\left(\psi_{c}, u_{c}, \varphi_{c}\right) & =\alpha \mathcal{R}\left(\tau_{\mathbf{a}}\left(\psi_{c}\right)\right)+\gamma\left(\tau_{\mathbf{a}}\left(\psi_{c}\right)-u_{c}\right) \tau_{\mathbf{a}}^{\prime}\left(\psi_{c}\right)-\left(\varphi_{c}\right)^{\top} \tau_{\mathbf{a}}^{\prime}\left(\psi_{c}\right), \\
\mathcal{F}_{3}(\sigma, u, z) & =\sum_{c \in\{r, g, b\}}\left(\kappa_{\mathcal{G}}^{\prime}(\sigma) * u_{c}\right)\left(\kappa_{\mathcal{G}}(\sigma) * u_{c}-z_{c}\right),
\end{aligned}
$$

where $\mathcal{R}$ is the derivative of the TV transform. We may find the solutions to $\mathcal{F}_{1}=0$ and $\mathcal{F}_{2}=0$ using gradient descent methods and the solution to $\mathcal{F}_{3}=0$ using the bisection method. The overall algorithm $\mathbb{A}_{1}$ is given in Algorithm 1.

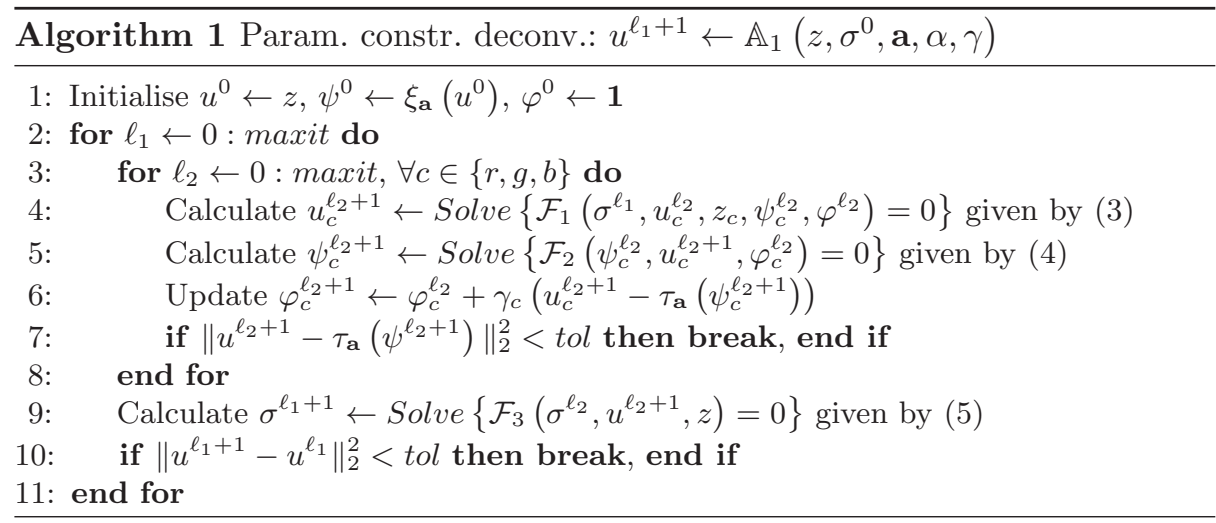

\subsection{Enhancement II - $\alpha$ Parameter Mask}

A mask is superimposed around the field of view in typical fundus images giving influence from an additional artificial boundary. This creates an issue particularly for the identification of the blur function. To address this, we may make use of a binary image mask $u_{\mathcal{G}} \in[0,1]^{m \times n}$ which has been graded by an expert to avoid heavy deconvolution in this region. We replace the scalar value $\alpha$ with a function $\Lambda$ which is dependent on the mask and given by

$$
\Lambda\left(\alpha, \rho ; u_{\mathcal{G}} ; \mathbf{x}\right)=\left[\kappa_{\mathcal{G}}(\rho) * \mathbb{P}_{\alpha}\left(u_{\mathcal{G}}\right)\right](\mathbf{x}), \quad \mathbb{P}_{\alpha}\left(u_{\mathcal{G}}\right)=\left\{\begin{array}{c}
\alpha \text { if } u_{\mathcal{G}}(\mathbf{x})=0 \\
u_{\mathcal{G}} \text { otherwise }
\end{array}\right.
$$


Note that, since we aim to avoid a discontinuous function $\Lambda$, we have used a convolution of a modified map with the kernel function $\kappa_{\mathcal{G}}(\rho)$.

\subsection{Enhancement III - Regularisation Parameter Selection}

We require a method of selecting the parameter $\alpha$ automatically. There exist techniques which may allow for the automatic selection of the regularisation parameter such as [5]. These techniques may be adapted to multi-dimensional $\alpha$ but they require information about the true image for comparison purposes. As an alternative, we may attempt to use blur (or sharpness) measures but these require a target sharpness level which is not known. In order to have an idea of what the image should look like, we would like to make use of vessel segmentation to measure accuracy of the deconvolution against an expert graded image.

Vessel Segmentation We now aim to provide a segmentation of the blood vessels in order to make a comparison with the graded map. We employ a simple method which will provide a reasonably accurate segmentation quickly. The method has three steps. First, we use contrast enhancement in order to make the vessels more easily distinguishable from the background. Next, we attempt to extract the vessels as the image foreground. Finally, we use thresholding in order to obtain a binary map of the blood vessels which may be compared with a grader's judgement. We begin by replacing the given image $u(\mathbf{x})$ with a contrast enhanced counterpart given by the mapping $\mathcal{C}_{p}: \mathbb{R}^{m \times n \times 3} \rightarrow \mathbb{R}^{m \times n \times 3}$, $\mathcal{C}_{p}(u(\mathbf{x}))=u^{\frac{1}{p}}(\mathbf{x})$, where the variable $p$ may be selected to give a more effective enhancement for vessel identification. In order to extract the vessels from the image, we blur the contrast enhanced image by a blur function $\kappa_{\mathcal{X}}$ and subtract the image. This enables the vessels to be identified and extracted as

$$
\mathcal{X}_{p}\left(u(\mathbf{x}), \kappa_{\mathcal{G}}(\theta, \mathbf{x})\right)=\left[\kappa_{\mathcal{G}}(\theta) * \mathcal{C}_{p}(u)\right](\mathbf{x})-\mathcal{C}_{p}(u(\mathbf{x})) .
$$

In order to distinguish the vessels from the background, we now require a binary image from $\mathcal{X}_{p}\left(u(\mathbf{x}), \kappa_{\mathcal{G}}(\theta, \mathbf{x})\right)$ which may be achieved by thresholding at a point $\mu \in \mathbb{R}$, which resembles implementation of a Heaviside function shifted by $\mu$. Since we aim to use this in a variational framework, we would like a continuous function to implement this step. We therefore use an approximation to the shifted Heaviside function given by $\mathcal{H}_{\varepsilon, \mu}(x)=\left(1+\exp \left(-2 \frac{x-\mu}{\varepsilon}\right)\right)^{-1}$, where $\varepsilon \in \mathbb{R}$ is a tuning parameter such that the limit of $\mathcal{H}_{\varepsilon, \mu}(x)$ as $\epsilon \rightarrow 0$ is the Heaviside function $H(x)$. The vessel map $\mathcal{V}$ may then be calculated from the given image as

$$
\mathcal{V}_{\varepsilon, \mu, p, \theta}(u(\mathbf{x}))=\mathcal{H}_{\varepsilon, \mu}\left(\mathcal{C}_{p}(u(\mathbf{x}))\right) \mathcal{X}_{p}\left(u(\mathbf{x}), \kappa_{\mathcal{G}}(\theta, \mathbf{x})\right)=\frac{\left[\left(\kappa_{\mathcal{G}}(\theta)-\delta\right) * \mathcal{C}_{p}(u)\right](\mathbf{x})}{1+\exp \left(-2 \frac{\mathcal{C}_{p}(u(\mathbf{x}))-\mu}{\varepsilon}\right)},
$$

where $\delta$ is the delta-function, which acts as the identity under convolution. 
Measuring Fitting We may measure the accuracy of segmentation using the Tannimoto Coefficient [6] and the Dice Similarity Coefficient [6] given by

$$
T_{c}(A, B)=\frac{\mathcal{N}(A \cap B)}{\mathcal{N}(A \cup B)} \quad \text { and } \quad D_{c}(A, B)=\frac{2 \mathcal{N}(A \cap B)}{\mathcal{N}(A)+\mathcal{N}(B)}
$$

respectively where $\mathcal{N}(A)$ denotes the number of elements in $A$. As the segmentation of the restored image $A$ tends towards the grader's segmentation $B$, both of the above error measures tend toward one.

\subsection{The Solution Algorithm}

In order to solve the resulting EL equations, we discretise the domain as

$$
\Omega=\left\{\left(c_{x} h_{x}, c_{y} h_{y}\right) \mid\left(c_{x}, c_{y}\right) \in \mathbb{Z}_{0}^{m} \times \mathbb{Z}_{0}^{n}\right\}, \quad \mathbb{Z}_{a}^{b}=\{c \in \mathbb{Z} \mid a \leq c \leq b-1\},
$$

where $h_{x}=(m-1)^{-1}$ and $h_{y}=(n-1)^{-1}$ are the step sizes in the $x$ and $y$ directions respectively. In order to gain speed by running in parallel, we split up the $m \times n$ image domain $\Omega$ into $M+2 r_{x} \times N+2 r_{y}$ blocks $\Omega_{i, j}$ where we allow an overlap of $r_{x}$ in each direction horizontally and of $r_{y}$ vertically. We may then process each of the $\mathcal{M N}$ blocks separately, where $\mathcal{M}=m / M$ and $\mathcal{N}=m / N$.

$$
\Omega_{i, j}=\left\{\left(M(i-1)+c_{x} h_{x}, N(j-1)+c_{y} h_{y}\right) \mid\left(c_{x}, c_{y}\right) \in \mathbb{Z}_{-r_{x}}^{M+r_{x}} \times \mathbb{Z}_{-r_{y}}^{N+r_{y}}\right\}
$$

defines the blocks for $i=1, \ldots, \mathcal{M}$ and $j=1, \ldots, \mathcal{N}$ and solve the problem for each simultaneously. The overall algorithm $\mathbb{A}_{2}$ is given in Algorithm 2.

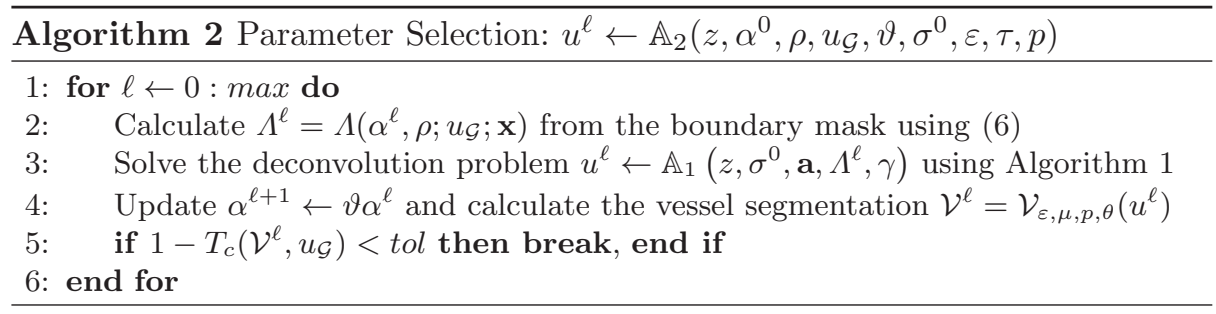

\section{Experimental Results}

In order to demonstrate the performance of this algorithm, we use a set of 20 blurred retinal scans, taken from the Digital Retinal Images for Vessel Extraction (DRIVE) data set which was obtained during a diabetic retinopathy screening program in the Netherlands. The set contains 20 test set and 20 training images, each of resolution 768x584 and acquired using a Canon CR5 non-mydriatic 3CCD camera with a 45 degree field of view. To test our algorithm, the images 
were blurred and processed on a Dell XPS 8700 with an Intel Core i7-4770 processor and 32GB RAM to facilitate efficient parallel computing which allows a 3x speedup over comparable tests run serially with 4GB RAM. The computation time for each deblurred image is approximately one minute. Improvement in the restored image is measured using Peak Signal-to-Noise Ratio (PSNR) [1] and Mean Squared Error (MSE) while $T_{c}$ measures the accuracy of the vessel segmentation. It can be noted in Figures 1 and 2 and in Table 1 that good results may be achieved using this method. In all test cases, the image is improved over the received data and the Tannimoto Coefficient corresponds well to PSNR.

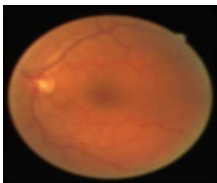

(a) $z$

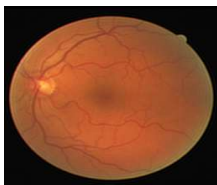

(b) $u$

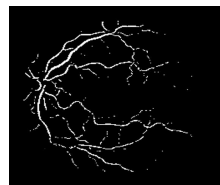

(c) $i_{\mathcal{V}}$

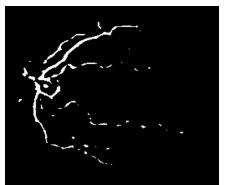

(d) $z_{\mathcal{V}}, T_{c}=0.4$

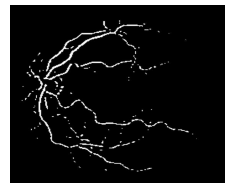

(e) $u_{\mathcal{V}}, T_{c}=0.7$

Fig. 1: Example of the restoration by Algorithm 2 of a retinal image corrupted by Gaussian blur and 1\% random noise. The restored image $u$ (b) is an improvement on the received image $z$ (a). The vessel segmentation of the restored image $u_{\mathcal{V}}$

(e) has improved considerably over the segmentation of the received image $z \mathcal{V}$

(d) and resembles more closely the target segmentation $i_{\mathcal{V}}$.

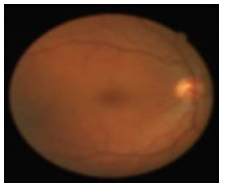

(a) $z$

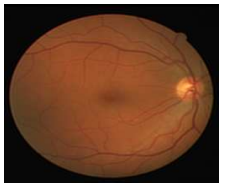

(b) $u$

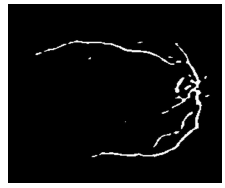

(c) $z_{\mathcal{V}}$

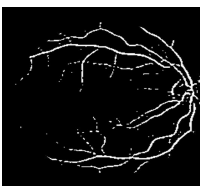

(d) $u \mathcal{v}$

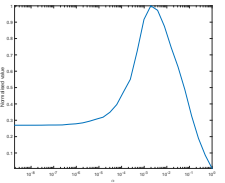

(e) $\alpha$ against $T_{c}$

Fig. 2: Example of the restoration by Algorithm 2 of a retinal image corrupted by Gaussian blur and random noise. The restored image $u(\mathrm{~b})$ is improved over $z$ (a). The segmentation has also improved, the Tannimoto Coefficient $\left(T_{c}\right)$ has risen from 0.34 to 0.71 . In (d), we can see the $\alpha$ selection determined by $T_{c}$.

\section{Conclusion}

In this paper, we have approached the problem of the deconvolution of retinal images using a new multi-channel implicitly constrained parametric framework. We have used available image masks to give a regularisation coefficient function which allows us to exclude artificial areas while using finite differences. We 


\begin{tabular}{l||ccc||ccc||ccc}
\multicolumn{1}{l||}{} & \multicolumn{3}{c||}{ Received Image $z$} & \multicolumn{3}{c||}{ Restored Image $u$} & \multicolumn{3}{c}{ Improvement } \\
Example & PSNR MSE & $T_{c}$ & PSNR & MSE & $T_{c}$ & PSNR & MSE & $T_{c}$ \\
\hline 5 & 33.06 & 32.16 & 0.3866 & 39.43 & 7.41 & 0.6467 & 6.37 & 24.75 & 0.2601 \\
\hline 16 & 32.01 & 40.89 & 0.4126 & 38.25 & 9.72 & 0.7012 & 6.24 & 31.16 & 0.2886 \\
\hline 18 & 29.90 & 66.59 & 0.3881 & 36.44 & 14.75 & 0.6409 & 6.55 & 51.84 & 0.2528 \\
\hline Average & 31.08 & 55.54 & 0.4153 & 36.76 & 14.70 & 0.6689 & 5.67 & 40.84 & 0.2536
\end{tabular}

Table 1: Table showing the error values of some test set images as well as the averages for the entire set. It can be seen that the PSNR and Tannimoto Coefficient have been improved in each case and the MSE has also decreased.

have also developed a regularisation parameter selection technique using vessel segmentation, which allows for the regularisation coefficient function to be set automatically without human intervention. We have presented results demonstrating the effectiveness of this new approach, which show an improvement in each blurred image and suggest that this technique is reliable for producing good-quality deconvolution results. In future research, we expect to improve the method by using new vessel segmentation methods $[8,9]$, optimising the code for computational efficiency, and evaluating the techniques on a large dataset. We may also improve on the illumination correction in our model with more advanced techniques and extend the model to other blur types.

\section{References}

1. Campisi, P., Egiazarian, K.: Blind Image Deconvolution. CRC Press (2007)

2. Chan, R.H., Tao, M., Yuan, X.: Constrained total variational deblurring models and fast algorithms based on alternating direction method of multipliers. SIAM J. Imaging Sci. 6(1), 680-697 (2013)

3. Chan, T.F., Wong, C.K.: Total variation blind deconvolution. IEEE T. Image Process. 7(3), 370-375 (1998)

4. Chen, K., Harding, S.P., Williams, B.M., Zheng, Y.: A new study of blind deconvolution with implicit incorporation of non-negativity constraints. International Journal of Computational Mathematics 2015, 860263 (2015)

5. Chen, K., Piccolomini, E.L., Zama, F.: An automatic regularization parameter selection algorithm in the total variation model for image deblurring. Numer. Algorithms pp. 1-20 (2013)

6. Crum, W.R., Camara, O., Hill, D.L.: Generalized overlap measures for evaluation and validation in medical image analysis. IEEE T. Med. Imaging 25(11), 1451-1461 (2006)

7. You, Y.L., Kaveh, M.M.: A regularization approach to joint blur identification and image restoration. IEEE T. Image Process. 5(3), 416-428 (1996)

8. Zhao, Y., Liu, Y., Wu, X., Harding, S.P., Zheng, Y.: Retinal vessel segmentation: An efficient graph cut approach with retinex and local phase. PLoS ONE 10, e0122332 (2015)

9. Zhao, Y., Rada, L., Chen, K., Harding, S., Zheng, Y.: Automated vessel segmentation using infinite perimeter active contour model with hybrid region information with application to retina images. IEEE T. Med. Imaging p. ePub (2015) 